\title{
Position does not affect the optic nerve sheath diameter during laparoscopy
}

\author{
Sang Hun Kim, Hyung Jin Kim, and Ki Tae Jung \\ Department of Anesthesiology and Pain Medicine, Chosun University Hostpital, Gwangju, Korea
}

\begin{abstract}
Background: Increase in intracranial pressure (ICP) is one of the physiologic changes during laparoscopic surgery, which is known to be associated with positional changes. Changes of ICP can be measured directly by invasive method, but ultrasonographic measurement of optic nerve sheath diameter (ONSD) is known to be a rapidly applicable technique for evaluating ICP. The aim of this study is to investigate the change of ONSD according to the positional change during laparoscopic surgery.

Methods: Female patients scheduled to undergo laparoscopic surgery were enrolled. Fifty-seven patients were assigned according to the position during surgery (Group T: gynecological surgery, Trendelenburg position, $\mathrm{n}=27$ vs. Group RT: laparoscopic cholecystectomy, Reverse trendelenburg position, $\mathrm{n}=30$ ). After induction of anesthesia, $\mathrm{ONSD}, \mathrm{PaCO}_{2}$, end-tidal carbon dioxide $\left(\mathrm{ETCO}_{2}\right)$, and mean arterial pressure (MAP) were measured. Parameters were measured at 6 time points during surgery.

Results: There were no significant differences in the demographic data of patients, procedure time, and anesthesia. After pneumoperitoneum and positional change, $\mathrm{ONSD}, \mathrm{ETCO}_{2}$, and $\mathrm{MAP}$ increased in both groups until 15 min and returned to the baseline. However, no significant differences in changes of ONSD, $\mathrm{PaCO}_{2}, \mathrm{ETCO}_{2}$, and $\mathrm{MAP}$ were observed between two groups.

Conclusions: ONSD during laparoscopic surgery with pneumoperitoneum increased slightly until 15 minutes, but there were no significant differences according to the position. Increases in ICP during laparoscopic surgery with short period of pneumoperitoneum would be small in disregard of position in patients without intracranial pathology.
\end{abstract}

Key Words: Intracranial pressure, Laparoscopy, Optic nerve, Trendelenburg position, Ultrasonography.

\section{Introduction}

In recent years, laparoscopic surgery has become the method of choice for many procedures which were previously performed

Corresponding author: Ki Tae Jung, M.D.

Department of Anesthesiology and Pain Medicine, Chosun University Hostpital, 365, Pilmun-daero, Dong-gu, Gwangju 501-717, Korea

Tel: 82-62-220-3223, Fax: 82-62-223-2333

E-mail: mdmole@chosun.ac.kr

Received: January 28, 2015.

Revised: February 26, 2015.

Accepted: March 31, 2015.

Korean J Anesthesiol 2015 August 68(4): 358-363

http://dx.doi.org/10.4097/kjae.2015.68.4.358 by open techniques because of it benefits with better cosmetic results, less postoperative pain and wound infection, earlier return to preoperative activities, etc. [1]. However, laparoscopic procedures require the establishment of artificial pneumoperitoneum which may lead to physiologic changes such as cardiovascular and respiratory compromise during pneumoperitoneum $[1,2]$. The impacts on the intracranial pressure (ICP) during laparoscopy are well-documented and several studies have demonstrated that the induction of artificial pneumoperitoneum provokes a measureable increase in ICP $[2,3]$. The mechanisms of increase of ICP during laparoscopy are suggested as follows: increase of intra-abdominal pressure, impairment of cerebrospinal fluid (CSF) absorption and impeded drainage of the lumbar venous plexus, increased pressure in the vascular compartment of sacral spaces, Trendelenburg position, and cerebral vasodila-

(c) This is an open-access article distributed under the terms of the Creative Commons Attribution Non-Commercial License (http://creativecommons.org/ licenses/by-nc/4.0/), which permits unrestricted non-commercial use, distribution, and reproduction in any medium, provided the original work is properly cited. 
tion due to hypercarbia [3].

Although the increase of ICP during laparoscopy may have possibility of a major cerebrovascular detriment, measurement of ICP is not implemented because the most reliable method for assessment of ICP is direct measurement of ICP via an invasive monitoring device such as an extraventricular drain and invasive ICP monitoring has a risk of severe complications with hemorrhage or infection [4]. However, assessing the optic nerve sheath diameter (ONSD) by transbulbar sonography ICP has recently been introduced as a useful tool for non-invasive detection of altered ICP [4,5]. Several studies have shown that measurement of ONSD is a quick and non-invasive method of detecting increased ICP and can be used as a strong and accurate predictor of increased ICP [5-8]. Despite the popularity of ultrasound measurement of the ONSD, this technique was used mainly for the emergency situation or critical care $[4,8]$.

A recent study on ultrasonographic measurement of ONSD during robot-assisted laparoscopic radical prostatectomy with steep Trendelenburg positioning revealed that ONSD increased approximately $12.5 \%$ and the increase of ICP corresponding to change of ONSD could be predicted [9]. However, no study on changes of ONSD according to the positional change during laparoscopic with short procedure time has been reported. We hypothesized that if ICP during laparoscopy is different according to the position, ONSD would likewise be different. Thus, we investigate the change of ONSD according to the positional change in laparoscopic surgery.

\section{Materials and Methods}

This study was approved by the Institutional Review Board and registered in ClinicalTrials.gov. A total of 60 patients scheduled to undergo laparoscopic gynecological surgery or laparoscopic cholecystectomy were enrolled after written informed consent was obtained. Only female patients who were American Society of Anesthesiologist class I to II and aged between 19 to 65 years were included in the study. Patients with previous neurologic diseases (e.g. hydrocephalus, intracranial hemorrhage, etc.) that may increase the ICP or patients with opthalmologic disease were excluded from enrollment. Patients were divided into two groups: Group T, laparoscopic gynecological surgery with Trendelenburg position (tilting the table down to an angle 30 degree) during surgery ( $\mathrm{n}=30)$; Group RT, laparoscopic cholecystectomy with reverse Trendelenburg position (tilting the table up to an angle 30 degree) during surgery $(\mathrm{n}=30)$.

Patients were premedicated with midazolam $0.05 \mathrm{mg} / \mathrm{kg}$ intramuscularly 30 minutes before the induction of anesthesia and transported to the operating room. Once in the operating room, patients were monitored with electrocardiogram, pulse oximetry $\left(\mathrm{SpO}_{2}\right)$, non-invasive arterial pressure (NIBP), and the bispectral index (BIS monitor A-2000; Aspect Medical Systems, Norwood, MA, USA). Anesthesia was induced with propofol (2 mg/kg), followed by administration of rocuronium bromide $(0.6 \mathrm{mg} / \mathrm{kg})$. After endotracheal intubation, mechanical ventilation was applied with 50\% oxygen-air mixture, using a tidal volume of 8-10 $\mathrm{ml} / \mathrm{kg}$ and adjusted to keep the peak inspiratory pressure below $30 \mathrm{cmH}_{2} \mathrm{O}$ during anesthesia. Respiratory rate of 10-12/min was applied and adjusted to maintain the end-tidal carbon dioxide $\left(\mathrm{ETCO}_{2}\right)$ levels between 35 and $40 \mathrm{mmHg}$ during surgery. A cannula was inserted to the radial artery for the continuous monitoring of arterial blood pressure and analysis of arterial blood gas. Anesthesia was maintained with desflurane and remifentanil infusion. The end-tidal concentration of desflurane was adjusted to maintain the BIS score within 40 to 60 and the targeted effect-site concentrations (Ce) of remifentanil were adjusted within 1.0 to $3.0 \mathrm{ng} / \mathrm{ml}$ using a TCI device (Orchestra ${ }^{\circledR}$ Base Primea, Fresenius Vial, France) based on the Marsh pharmacokinetic model to keep the changes of vital signs within $20 \%$ of baseline value.

About 5 minutes after induction of anesthesia, when stabilization of cardiovascular status was achieved, baseline ONSD was measured in the supine position by ulrasonographic measurement. A thick gel layer was applied to the closed upper eyelids of patients and the linear 13-6 MHz probe (M-Turbo; Fujifilm Sonosite Inc., Tokyo, Japan) was carefully placed on the gel. Excessive pressure was avoided with gentle handling and the probe was adjusted to display the entry of the optic nerve into the globe in the two-dimensional mode. After obtaining optimal contrast between the retrobulbar echogenic fat tissue and the vertical hypoechoic band, the ONSD was measured $3 \mathrm{~mm}$ behind the optic disc using an electronic caliper. The final ONSD value was calculated by averaging 4 measured values: measured values of transverse and sagittal plane of both eyes.

After measurement of baseline ONSD, artificial pneumoperitoneum was made by the surgeon. The insufflation pressure during the procedure was limited to $12 \mathrm{mmHg}$. Then, the patient's position was changed for the surgery. Trendelenburg position with an angle of 30 degree was applied in the patients in group $\mathrm{T}$ who underwent laparoscopic gynecological surgery, while reverse Trendelenburg position with an angle of 30 degree was applied in the patients of group RT who underwent laparoscopic cholecystectomy.

About 5 minutes after pneumoperitoneum and position change were achieved, ONSD was measured in the same way with time interval. When the measurement of ONSD was performed, $\mathrm{ETCO}_{2}$ and mean arterial pressure (MAP) were also measured simultaneously. Measurements were totally performed at 6 time-points: T0, before introducing pneumoperitoneum; T1, 5 minutes after introducing pneumoperitoneum and position change; T2, 10 minutes after introducing pneumoperitoneum 
and position change; T3, 15 minutes after introducing pneumoperitoneum and position change; T4, 30 minutes after introducing pneumoperitoneum and position change; T5, 5 minutes after discontinuing pneumoperitoneum and the patient's position had returned to supine. $\mathrm{PaCO}_{2}$ was measured at time-point of T0, T3, T4, and T5. If the measured ONSD was more than 5 $\mathrm{mm}$, it was regarded as significant increase of ICP which is more than $20 \mathrm{cmH}_{2} \mathrm{O}[9,10]$, and the number of patients with ONSD more than $5 \mathrm{~mm}$ was counted. We also measured the procedure time with laparoscopy during operation, which is defined as the time from initiation of artificial pneumoperitoneum to end of pneumoperitoneum, and total times of anesthesia.

Sample size was calculated with two-tailed significance level ( $\alpha=0.05$ ) and 90\% power. Effect size was calculated on the basis of a previous study which showed ONSD of normal adults as 4.60 $\pm 0.41 \mathrm{~mm}$ [8]. If the cut-off value of increased ICP is regarded as $5.0 \mathrm{~mm}$ [10], then the effect size was 0.976 . Required sample size was 24 patients in each group, and a total of 60 patients were enrolled in due consideration of drop-out.

Data were analyzed using SPSS (Windows ver. 12.0, SPSS Inc., Chicago, IL, USA) and all results are presented as mean \pm

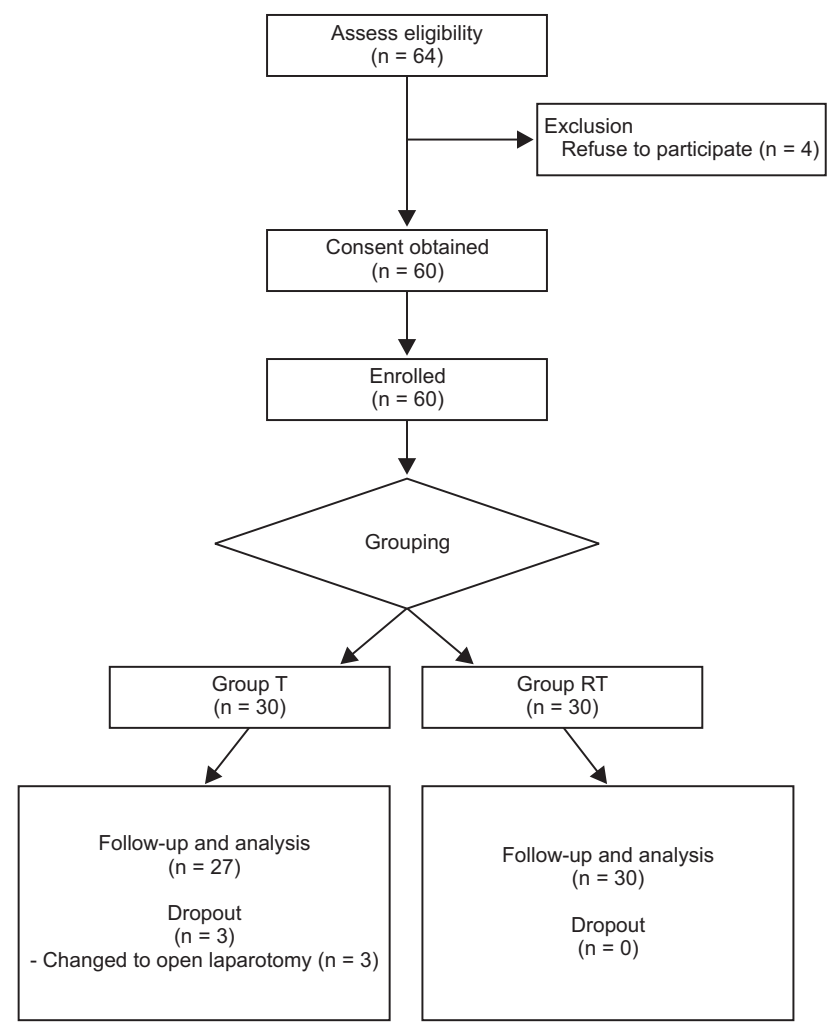

Fig. 1. CONSORT flow diagram for the study. Group T: laparoscopic gynecological surgery with Trendelenburg position during surgery, Group RT: laparoscopic cholecystectomy with reverse Trendelenburg position during surgery $(\mathrm{n}=30)$. standard deviation. Statistical analysis was performed using ttest, Fisher's exact test, and chi-square tests to evaluate statistical significance between the two groups for age, weight, height, duration of laparoscopic procedure, and duration of anesthesia. Repeated measures of ANOVA were performed to compare the differences of $\mathrm{MAP}, \mathrm{ETCO}_{2}, \mathrm{PaCO}_{2}$, and $\mathrm{ONSD}$ between groups. If there were overall differences within the groups, t-test with Turkey's test was performed for the post hoc analysis. Values with $\mathrm{P}<0.05$ were considered statistically significant.

\section{Results}

A total of 64 patients were recruited for the study and 60 patients were finally enrolled (Fig. 1). Three patients were dropped out in group $\mathrm{T}$ because laparoscopic operation was converted to the open laparotomy. There were no significant differences in the demographic data of patients, procedure time, and anesthesia (Table 1).

After pneumoperitoneum and positional change, MAP, and $\mathrm{ETCO}_{2}$ increased in both groups $\left(\mathrm{P}<0.001\right.$ in $\mathrm{MAP}$ and $\mathrm{ETCO}_{2}$, Figs. 2 and 3). However, no significant differences in changes of MAP and $\mathrm{ETCO}_{2}$ were observed between two groups $(\mathrm{P}=0.868$ in MAP; $\mathrm{P}=0.226$ in $\mathrm{ETCO}_{2}$ ). After 15 minutes from pneumoperitoneum and positional change, $\mathrm{MAP}$, and $\mathrm{ETCO}_{2}$ returned to the baseline level in both groups. There were no significant differences in $\mathrm{PaCO}_{2}$ level during measurement period in both groups ( $\mathrm{P}=0.214$, Fig. 3 ).

The mean values of ONSD increased after pneumoperitoneum and positional change $(\mathrm{P}<0.001$, Fig. 4). Such increases lasted until minutes and returned to similar levels after 15 minutes. However, no significant differences in the changes of ONSD were observed between two groups $(P=0.630)$. The number of patients with ONSD more than $5 \mathrm{~mm}$ also showed no significant differences $(\mathrm{P}=0.887 ; 5$ in group $\mathrm{T}$ vs 6 in group RT).

Table 1. Demographic Variables and the Number of Patients with Significant Increase of ONSD

\begin{tabular}{lccc}
\hline & $\begin{array}{c}\text { Group T } \\
(\mathrm{n}=27)\end{array}$ & $\begin{array}{c}\text { Group RT } \\
(\mathrm{n}=30)\end{array}$ & $\mathrm{P}$ \\
\hline Age $(\mathrm{yr})$ & $43.9 \pm 8.9$ & $46.1 \pm 9.8$ & 0.349 \\
Weight $(\mathrm{kg})$ & $60.6 \pm 10.9$ & $59.8 \pm 13.0$ & 0.122 \\
Height $(\mathrm{cm})$ & $159.6 \pm 5.9$ & $156.9 \pm 7.9$ & 0.577 \\
ASA class (I/II) & $18 / 9$ & $19 / 11$ & 0.977 \\
Duration of operation (min) & $39.1 \pm 2.9$ & $36.7 \pm 2.0$ & 0.088 \\
Duration of anesthesia (min) & $80.6 \pm 4.7$ & $78.9 \pm 4.0$ & 0.668 \\
ONSD $>5$ mm (n) & 5 & 6 & 0.846 \\
\hline
\end{tabular}

Values are expressed as mean \pm SD or number. No significant differences in demographic variables were observed among the groups. ASA: American Society of Anesthesiologists. ONSD: optic nerve sheath diameter. 


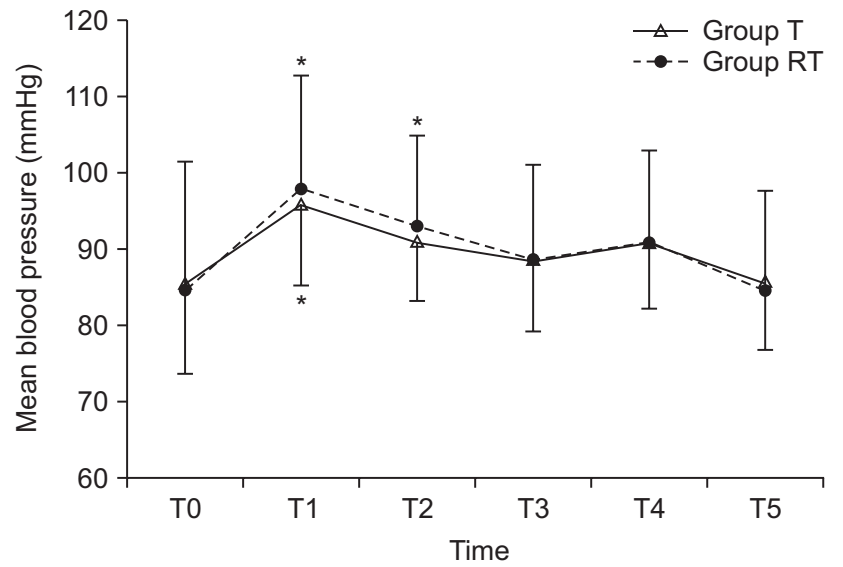

Fig. 2. Changes of mean arterial pressure during laparoscopic surgery. Group T: laparoscopic gynecological surgery with Trendelenburg position during surgery, Group RT: laparoscopic cholecystectomy with reverse Trendelenburg position during surgery. T0: baseline (before introducing pneumoperitoneum), T1: 5 minutes after introducing pneumoperitoneum and positional change, T2: 10 minutes after introducing pneumoperitoneum and positional change, T3: 15 minutes after introducing pneumoperitoneum and positional change, T4: 30 minutes after introducing pneumoperitoneum and positional change, T5: 5 minutes after discontinuing pneumoperitoneum and the patient's position has returned to supine. ${ }^{*} \mathrm{P}<0.05$ compared with baseline value at $\mathrm{T} 0$.

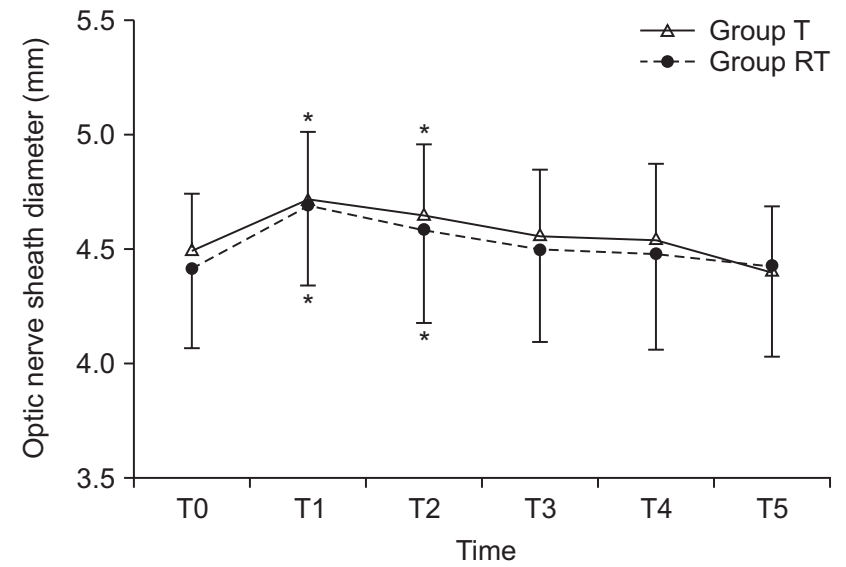

Fig. 4. Changes of optic nerve sheath diameter during laparoscopic surgery. Group T: laparoscopic gynecological surgery with Trendelenburg position during surgery, Group RT: laparoscopic cholecystectomy with reverse Trendelenburg position during surgery. T0: baseline (before introducing pneumoperitoneum), T1: 5 minutes after introducing pneumoperitoneum and positional change, T2: 10 minutes after introducing pneumoperitoneum and positional change, T3: 15 minutes after introducing pneumoperitoneum and positional change, T4: 30 minutes after introducing pneumoperitoneum and positional change, T5: 5 minutes after discontinuing pneumoperitoneum and the patient's position has returned to supine. $* \mathrm{P}<0.05$ compared with baseline value at T0.
A

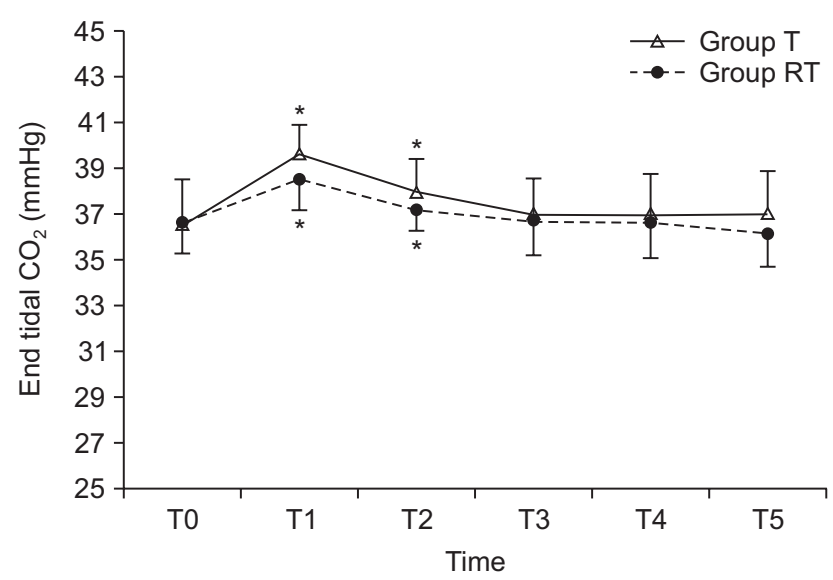

B

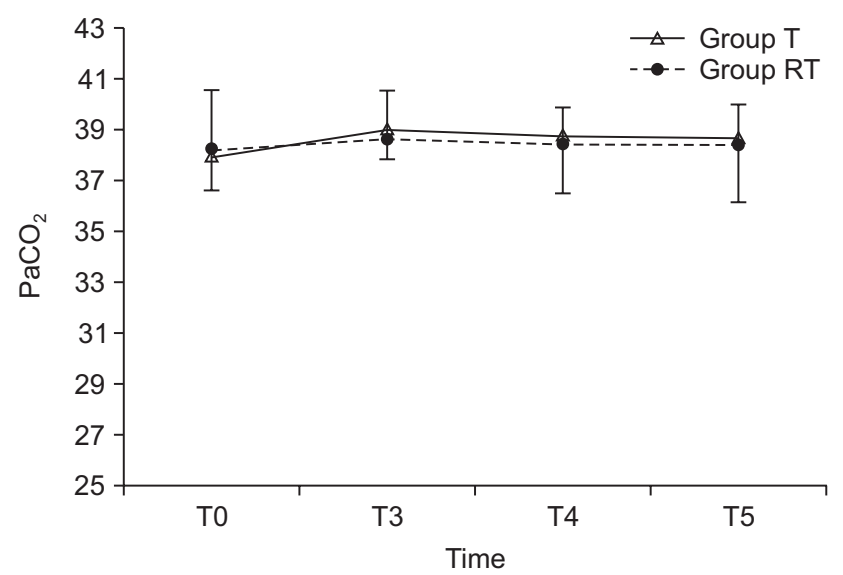

Fig. 3. Changes of end-tidal $\mathrm{CO}_{2}(\mathrm{~A})$ and $\mathrm{PaCO}_{2}$ (B) during laparoscopic surgery. Group T: laparoscopic gynecological surgery with Trendelenburg position during surgery, Group RT: laparoscopic cholecystectomy with reverse Trendelenburg position during surgery. T0: baseline (before introducing pneumoperitoneum), T1: 5 minutes after introducing pneumoperitoneum and positional change, T2: 10 minutes after introducing pneumoperitoneum and positional change, T3: 15 minutes after introducing pneumoperitoneum and positional change, T4: 30 minutes after introducing pneumoperitoneum and positional change, T5: 5 minutes after discontinuing pneumoperitoneum and the patient's position has returned to supine. ${ }^{*} \mathrm{P}<0.05$ compared with baseline value at $\mathrm{T} 0$.

\section{Discussion}

In the current study, we assessed the change of ONSD according to the positional changes (Trendelenburg and reverse Trendelenburg) during laparoscopic surgery. We found no significant differences in the changes of ONSD despite position.
Measurement of ONSD is a non-invasive and indirect method to determine the changes of ICP, which requires validation of correlation between OSND and ICP. Kimberly, who studied the correlation of ultrasound measurements of ONSD and direct measurements of ICP with ventriculostomy [11], systematically confirmed the significant positive correlation between two 
methods with sensitivity of $88 \%$ and specificity of $93 \%$. A recent study that evaluated the efficacy of sonographic ONSD in estimating ICP showed that ONSD can be considered as a strong and accurate predicting factor for increased ICP with sensitivity and specificity of $100 \%$ [8]. In the current study, we used the cut-off value of increased ICP as $5.0 \mathrm{~mm}$ based on a previous study which estimated a sensitivity of $100 \%$ for an ONSD of 5 $\mathrm{mm}$ in predicting the ICP more than $20 \mathrm{cmH}_{2} \mathrm{O}$ [10]. With this basis, we hypothesized that measurement of ONSD during laparoscopy can predict the changes of ICP.

Several trials were conducted to evaluate the changes of ICP during laparoscopy with ONSD $[9,12]$. Kim et al. [9] reported that $\mathrm{CO}_{2}$ pneumoperitoneum with steep Trendelenburg positioning in patients undergoing robot-assisted laparoscopic radical prostatectomy (RALRP) increased the ONSD approximately $12.5 \%$ in 10 and 30 minutes after 30-degree Trendelenburg positioning with pneumoperitoneum, thus the increase of ICP could be predicted. These results were in agreement with ours that also showed a significant increase in ONSD 5 and 10 minutes after pneumoperitoneum and positional changes (Group T, 4.7\% in $\mathrm{T} 1$ and $3.4 \%$ in T2; Group RT, 6.3\% in T1 and 3.9\% in T2). However, in the current study there are two remarkable differences compared to Kim's study. First, ONSD measured 30 minutes after pneumoperitoneum and positional changes showed no significant differences compared to baseline. Second, the rate of changes in ONSD was smaller in our study (6.3 vs $12.5 \%$ in maximum changes), suggesting that there can be temporary increase in ICP during pneumoperitoneum, but it became normalized quickly. Verdonck et al. [12] reported that ONSD remains constant during RALRP, increases in ICP are likely to be small because of compensation mechanisms for increase in intracranial blood volume. They explained such compensation mechanisms as follows. Dynamic equilibrium of parenchymal tissue, arterial and venous blood, and CSF according to the Monroe-Kellie doctrine can control the ICP dramatically [12,13]. Because increase in volume of one compartment must be compensated by decreases in volume of the others [13], increase of the intracranial blood volume by pneumoperitoneum and head down position has compensated by decrease of CSF in the same amount, and the ICP change would not occur [12]. Moreover, the human brain can also translocate CSF very rapidly so that ICP can be regulated quickly [14]. According to an animal study, when the ICP has increased, the CSF can be infused intrathecally by a rate of $2 \mathrm{ml} / \mathrm{min}$ to maintain the ICP at $20 \mathrm{mmHg}$ [14]. Thus, increased blood volume during RALRP can rapidly be compensated by an efflux of CSF [12]. Likewise, in this study intracranial blood volume changed due to pneumoperitoneum, positional change, and increased MAP during time-point of T1 and T2 and ONSD might have increased at that time-point in this study. However, in this study we allocated only patients without intracranial pathology, meaning that adequate compensation mechanisms may have worked in the subjects. Thus, in our study even the ONSD increased, the maximum rate of changes in ONSD was $6.36 \%$, which was relatively small. It seems that the compensation mechanisms of brain in ICP are important reasons for this small rate of change in the ONSD.

The unique results of this study are that there were no differences in changes of ONSD according to the position. The ONSD showed similar changes in both Trendelenburg and reverse Trendelenburg group. This also suggests that ICP can be maintained in disregard of position and the increases in ICP can be sustained to a small extent. It seems that the same compensatory mechanism as mentioned above can explain these results [12,13]. One of the main differences of our study compared to the previous studies is the duration of pneumoperitoneum [12,15]. The procedure time of the current study was only less than 40 minutes, which was much shorter. Even though ICP was raised consistently during laparoscopic surgery, compensation mechanisms do not become exhausted up to a point where the ONSD increases [12]. In addition, there were no significant differences in $\mathrm{ETCO}_{2}$ and $\mathrm{PaCO}_{2}$ between two groups. Considering that the ONSD changes rapidly in response to $\mathrm{ETCO}_{2}$ and has benefits to close ICP monitoring in response to $\mathrm{CO}_{2}$ change [9], these results suggest that increases in ICP during laparoscopic surgery with short period of pneumoperitoneum would be small in disregard of position.

There are some limitations in this study. First, we included only female patients to match the demographic data because we defined the group RT as laparoscopic gynecologic surgery. Thus, these results cannot represent the whole population. Second, we did not measure $\mathrm{PaCO}_{2}$ at the time-point of $\mathrm{T} 1$ and $\mathrm{T} 2$, which was a methodological error in the planning of the study. At first, we were not concerned with the possibility of rapid changes of $\mathrm{PaCO}_{2}$ according to the $\mathrm{ETCO}_{2}$ and omitted the measurement of $\mathrm{PaCO}_{2}$ at that time-point. Considering that the $\mathrm{PaCO}_{2}$ is strongly associated with the changes of ICP and ONSD, it was an obvious error. However, we collected $\mathrm{ETCO}_{2}$ data at the same time-point with ONSD, and we could see the changes of ONSD in response to $\mathrm{ETCO}_{2}$ as in a previous study [15]. However, further evaluation remains. Third, although increase in ICP can be an important physiologic change during laparoscopy, complications due to elevated ICP is a relatively rare event, and significant increase of ICP due to position may be rare itself in the clinical situation [12]. In addition, cut-off value of ONSD associated with ICP is not well defined, and may differ according to the typical characteristics of the population [6-8,10-12,15]. Thus, absence of increase in ONSD cannot fully guarantee the safety of all patients.

In conclusion, ONSD during laparoscopic surgery with pneumoperitoneum increased slightly until 15 minutes, but 
there were no significant differences according to the position. Increases in ICP during laparoscopic surgery with short period of pneumoperitoneum would be small in disregard of position in patients without intracranial pathology.

\section{Acknowledgments}

This study was supported by research fund from Chosun University, 2013.

\section{References}

1. Gonzalez R, Smith CD, McClusky DA 3rd, Ramaswamy A, Branum GD, Hunter JG, et al. Laparoscopic approach reduces likelihood of perioperative complications in patients undergoing adrenalectomy. Am Surg 2004; 70: 668-74.

2. Grabowski JE, Talamini MA. Physiological effects of pneumoperitoneum. J Gastrointest Surg 2009; 13: 1009-16.

3. Halverson A, Buchanan R, Jacobs L, Shayani V, Hunt T, Riedel C, et al. Evaluation of mechanism of increased intracranial pressure with insufflation. Surg Endosc 1998; 12: 266-9.

4. Geeraerts T, Merceron S, Benhamou D, Vigue B, Duranteau J. Non-invasive assessment of intracranial pressure using ocular sonography in neurocritical care patients. Intensive Care Med 2008; 34: 2062-7.

5. Bauerle J, Schuchardt F, Schroeder L, Egger K, Weigel M, Harloff A. Reproducibility and accuracy of optic nerve sheath diameter assessment using ultrasound compared to magnetic resonance imaging. BMC Neurol 2013; 13 : 187.

6. Legrand A, Jeanjean P, Delanghe F, Peltier J, Lecat B, Dupont H. Estimation of optic nerve sheath diameter on an initial brain computed tomography scan can contribute prognostic information in traumatic brain injury patients. Crit Care 2013; 17: R61.

7. Hightower S, Chin EJ, Heiner JD. Detection of increased intracranial pressure by ultrasound. J Spec Oper Med 2012; 12: 19-22.

8. Amini A, Kariman H, Arhami Dolatabadi A, Hatamabadi HR, Derakhshanfar H, Mansouri B, et al. Use of the sonographic diameter of optic nerve sheath to estimate intracranial pressure. Am J Emerg Med 2013; 31: 236-9.

9. Kim MS, Bai SJ, Lee JR, Choi YD, Kim YJ, Choi SH. Increase in intracranial pressure during carbon dioxide pneumoperitoneum with steep trendelenburg positioning proven by ultrasonographic measurement of optic nerve sheath diameter. J Endourol 2014; 28: 801-6.

10. Tayal VS, Neulander M, Norton HJ, Foster T, Saunders T, Blaivas M. Emergency department sonographic measurement of optic nerve sheath diameter to detect findings of increased intracranial pressure in adult head injury patients. Ann Emerg Med 2007; 49 : 508-14.

11. Kimberly HH, Shah S, Marill K, Noble V. Correlation of optic nerve sheath diameter with direct measurement of intracranial pressure. Acad Emerg Med 2008; 15: 201-4.

12. Verdonck P, Kalmar AF, Suy K, Geeraerts T, Vercauteren M, Mottrie A, et al. Optic nerve sheath diameter remains constant during robot assisted laparoscopic radical prostatectomy. PLoS One 2014; 9: e111916.

13. Eklund A, Smielewski P, Chambers I, Alperin N, Malm J, Czosnyka M, et al. Assessment of cerebrospinal fluid outflow resistance. Med Biol Eng Comput 2007; 45: 719-35.

14. Kalmar AF, De Ley G, Van Den Broecke C, Van Aken J, Struys MM, Praet MM, et al. Influence of an increased intracranial pressure on cerebral and systemic haemodynamics during endoscopic neurosurgery: an animal model. Br J Anaesth 2009; 102: 361-8.

15. Kim JY, Min HG, Ha SI, Jeong HW, Seo H, Kim JU. Dynamic optic nerve sheath diameter responses to short-term hyperventilation measured with sonography in patients under general anesthesia. Korean J Anesthesiol 2014; 67: 240-5. 